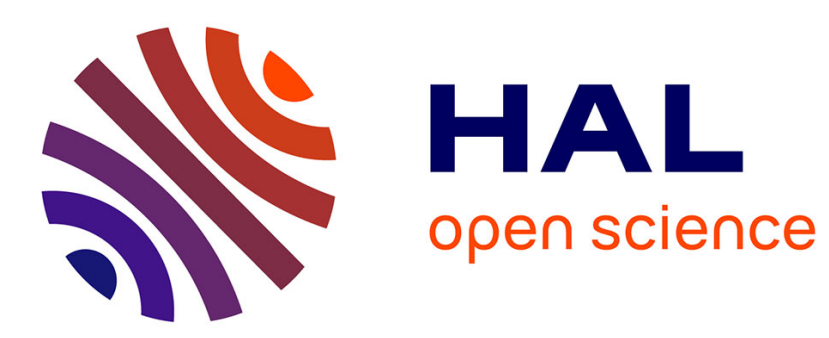

\title{
CALCULATION OF NUCLEAR QUADRUPOLE INTERACTION IN NON-CUBIC METALS AND ALLOYS
}

\author{
M. Piecuch, Ch. Janot
}

\section{- To cite this version:}

M. Piecuch, Ch. Janot. CALCULATION OF NUCLEAR QUADRUPOLE INTERACTION IN NONCUBIC METALS AND ALLOYS. Journal de Physique Colloques, 1976, 37 (C6), pp.C6-359-C6-369. 10.1051/jphyscol:1976668 . jpa-00216779

\section{HAL Id: jpa-00216779 https://hal.science/jpa-00216779}

Submitted on 1 Jan 1976

HAL is a multi-disciplinary open access archive for the deposit and dissemination of scientific research documents, whether they are published or not. The documents may come from teaching and research institutions in France or abroad, or from public or private research centers.
L'archive ouverte pluridisciplinaire $\mathbf{H A L}$, est destinée au dépôt et à la diffusion de documents scientifiques de niveau recherche, publiés ou non, émanant des établissements d'enseignement et de recherche français ou étrangers, des laboratoires publics ou privés. 


\title{
CALCULATION OF NUCLEAR QUADRUPOLE INTERACTION IN NON-CUBIC METALS AND ALLOYS
}

\author{
M. PIECUCH and Ch. JANOT \\ Laboratoire de Physique du Solide (L. A. ${ }^{\circ}$ 155) \\ Université de Nancy-I, C. O. no 140, 54037 Nancy-Cedex, France
}

\begin{abstract}
Résumé. - Cet article présente un calcul général de la contribution locale au gradient de champ électrique dans les métaux et alliages dilués de transition non cubiques, ainsi que sur une impureté de transition dans un métal normal non cubique. En prenant en compte l'influence de la température et les effets à $\mathrm{N}$-corps, une théorie dynamique conduit, dans l'approximation de la phase aléatoire, à l'expression de la valeur moyenne de tout opérateur. L'influence de l'anisotropie respectivement sur les intégrales de dérive et les intégrales de recouvrement est clairement mise en évidence.
\end{abstract}

\begin{abstract}
This article deals with a general calculation of the localized contribution to the electric field gradient in transition non-cubic metals and alloys, and on transition impurity in normal non-cubic metals. Taking into account temperature and many-body effects, a first order perturbation dynamical theory, in the random phase approximation leads to the mean value of any operator. The influence of the material anisotropy on both crystal field and transfer integrals is analyzed in details.
\end{abstract}

1. Introduction. - A compilation [1] of experimental results in the field of nuclear quadrupole interactions in metals and alloys has been recently published and it contains a wealth of interesting data, as well as evidences of the increasing interest for the understanding of the factors that influence these interactions.

However, from a theoretical point of view, many things are far from being clearly explained. For instance, if we concentrate on the hexagonal closepacked metals and alloys, as reported in our previous works $[2,3]$, the signs, the temperature and material dependences of the EFG have not been completely interpreted by now, except perhaps in the case of the normal pure hcp metal (Be, $\mathrm{Mg}, \mathrm{Cd}, \mathrm{Zn}$ ) for which DAS [4] achieved a satisfactory approach in an OPW description of the band structure, or in the case of transition impurities in these metals when virtual bound 3d states can be treated in the Hartree-Fock approximation $[2,3]$. It is not the purpose of the paper to return to an extensive critical survey of the others many theoretical approachs of the EFG in metals, since it has been already done very often $[2,3,4]$. Let us just recall that the main problem is to elucidate the relative contribution of electrons and lattice to the EFG. The lattice contribution (positive ions plus a uniform background of free electron gas) is usually obtained through summation procedure $[5,6,3]$; but it is rather easy to see $[7,8]$ that the final value obtained for $e q_{\mathrm{NLoc}}$ changes drastically when the Coulomb potential of the bare ions is replaced by more realistic ones as Thomas-Fermi, oscillating Friedel or Hartree potential. So even this mere point of the lattice contribution cannot be considered as well settled. The localized (or electronic) contribution to the EFG can be expressed as :

$$
\begin{aligned}
e q_{\mathrm{Loc}} & =\left\langle\psi_{n k}(r)\left|\frac{3 \cos ^{2} \theta-1}{r^{3}}\right| \psi_{n k}(r)\right\rangle \\
& =\left\langle\psi_{n k}(r)\left|\frac{2 P_{2}^{0}(\cos \theta)}{r^{3}}\right| \psi_{n k}(r)\right\rangle
\end{aligned}
$$

where $n$ refers to the bands and $k$ to the states in each band, lying below the Fermi surface. It would be worthwhile obtaining the value of $e q_{\text {Loc }}$ because it is an indirect genuine measurement of the space distribution of the electrons in anisotropic material, which could be a palliative observation to the direct study of the equipotential lines in these materials. In fact, it is not a trivial question, since one needs the knowledge of the wave functions for all the occupied Fermi volume, and solutions can only be achieved through drastic approximations. In addition to the OPW approach in normal hcp metals made by Das [4] or Mohapatra et al. [9], another model has been proposed [10] by Watson, Gossard and Yafet (WGY model in the following) for the pure transition metals; in this model the surface of a Fermi sphere is distorted by the crystal potential (which is merely reduced to its $l=2$ component) and this results in an expression of the local EFG involving only the density of states at the Fermi surface. However the WGY analysis, not only has not considered the eventual Fermi volume effects, but in addition has left out of count a contri- 
bution (called $F_{0}$ in their paper) to the Coulomb integrals whose effects would have been to enhance the polarization of the electrons by the crystal potential.

As a logical extension to our previous works [2, 3], the purpose of this paper is to present a general calculation of the local EFG taking into account the temperature and dynamical effects, in hep transition metals and alloys and on transition impurities in hcp normal metals.

2. General features of the calculation. - As already mentioned, obtaining quantitative results for the local EFG needs accurate electronic wave-functions which, unfortunately, are not available for most of the non-cubic metals and alloys. Among approximation process, a procedure consists of writing the oneelectron Hamiltonian and then taking a convenient description (APW, KKR, etc.) for the wave functions, so leading to the complete study of the system band structure. Such a procedure has been used by Demangeat [11] to obtain the EFG arising from the spinorbit coupling in ferromagnetic nickel.

The purpose of these papers is less to provide numerical result for a particular system than to give a general analysis which could be valid for qualitative interpretation in the most various experimental situation.

So, our prospect is to analyze the influence of anisotropy on the electrons in a metal ; to be more precise, this calculation consists of giving the mean value of a time-depending operator $\hat{A}(t)$ whose basic properties are the following:

$-\langle\hat{A}(t)\rangle=0$ for cubic metal or isotropic environment.

$-\langle\hat{A}(t)\rangle$ depends mainly on only one electronic band (nd for transition metals).

- $\hat{A}(t)$ is localized so that the matrix elements of $\hat{A}$ can be expressed only with wave functions centered on one site

- $\widehat{A}(t)$ is not spin dependent.

The EFG operator $\frac{2 P_{2}^{0}(\cos \theta)}{r^{3}}$ satisfies these basic properties.

Then, the metal anisotropy will be considered as a weak perturbation with respect to a cubic environment, which is a rather consistent hypothesis for hep structure having $c / a$ ratio not too far from the ideal value, and small energy difference with CFC structures.

With the restrictive properties of the $\hat{A}(t)$ operator, the problem of its mean value can be expressed in the tight-binding model over a set of functions [11] including $s$-band like functions $|\mathbf{k} s \sigma\rangle$ and $l$-like atomic states $|m \lambda \sigma\rangle$ centered on the site $\lambda$. As explained in an earlier paper [3], the s-band structure and the $l-s$ interactions are second order in anisotropy dependence of $\widehat{A}(t)$, and consequently will be neglected in the following.

Assuming that the crystal field anisotropy can be considered as a perturbation, the Hamiltonian will be written :

$$
\mathfrak{H}=\mathfrak{H}_{0}+\mathfrak{H}_{1}
$$

where $\mathscr{H}_{0}$ describes an isotropic metal quite near to the actual one ; in particular, it contains s-band energy, the $s-l$ mixing interaction and the two-body parts of electron-electron interaction.

Over the $|k s \sigma\rangle$ and $|m \lambda \sigma\rangle$ states, $\mathscr{H}_{1}$ can be written :

$$
\mathscr{H}_{1}=\sum_{m \sigma \lambda} V_{m}^{\lambda} C_{m \sigma \lambda}^{+} C_{m \sigma \lambda}+\sum_{m \sigma \lambda \lambda^{\prime}} \Delta T_{m}^{\lambda \lambda^{\prime}} C_{m \sigma \lambda}^{+} C_{m \sigma \lambda^{\prime}}
$$

where $V_{m}^{\lambda}$ is the change due to anisotropy in the crystal field integrals centered on the $\lambda$ site and $\Delta T_{m}^{\lambda \lambda^{\prime}}$ is the change due to anisotropy in the transfer integrals between $\lambda$ and $\lambda^{\prime}$ sites. Here is introduced the extrahypothesis that the anisotropy dependence of the transfer integrals gave only diagonal perturbation terms; this assumption is not so obvious and is mainly introduced for simplicity's sake.

The $\hat{A}(t)$ operator can be written as well :

$$
\widehat{A}(t)=\sum_{m m^{\prime} \sigma} A_{m m^{\prime}}^{\lambda} C_{m \sigma \lambda}^{+} C_{m^{\prime} \sigma \lambda}
$$

with

$$
A_{m m^{\prime}}^{\lambda}=\left\langle\lambda m \sigma|\hat{A}| \lambda m^{\prime} \sigma\right\rangle
$$

and $\langle\hat{A}(t)\rangle$ can be calculated using linear response theory, i. e. :

$$
<\widehat{A}(t)>=\operatorname{Tr}\{\hat{\rho} \hat{A}\}
$$

where $\hat{\rho}$ is the density matrix corresponding to the Hamiltonian and which obeys to the equation of motion :

$$
i \frac{\mathrm{d} \hat{\rho}}{\mathrm{d} t}=\left[\mathscr{H}_{0}+\mathscr{H}_{1}, \hat{\rho}\right]
$$

Using the properties of $\hat{A}$ and $\mathscr{H}$ and going through simple mathematics, this leads to

$$
<\widehat{A}(t)>=i \operatorname{Tr}\left\{\int_{-\infty}^{t} \rho_{0}\left[\widehat{A}\left(t-t^{\prime}\right), \mathcal{H}_{1}\left(t^{\prime}\right)\right] \mathrm{d} t^{\prime}\right\}
$$

The combination of eq. (2), (3) and (4) gives the following expression for $<\widehat{A}(t)>$

$$
\langle\hat{A}(t)\rangle=\sum_{n \lambda m^{\prime} m^{\prime} \sigma^{\prime}}\left\{\int_{-\infty}^{+\infty} \sum_{m \sigma} K_{1}\left(t-t^{\prime}\right) A_{m^{\prime} m^{m}}^{\mu} V_{m}^{\lambda} \mathrm{d} t^{\prime}+\int_{-\infty}^{+\infty} \sum_{\lambda^{\prime} m \sigma} K_{2}\left(t-t^{\prime}\right) A_{m^{\prime} m^{*}}^{\mu} \Delta T_{m}^{\lambda \lambda^{\prime}} \mathrm{d} t^{\prime}\right\}
$$


where $K_{1}$ and $K_{2}$ are shortened expressions for the following so-called response functions :

$$
\begin{gathered}
K_{1}\left(\mu \lambda m m^{\prime} m^{\prime \prime} \sigma \sigma^{\prime} \mid t\right)=-i \theta(t)\left\{\operatorname{Tr} \rho_{0}\left[C_{\mu m^{\prime} \sigma^{\prime}}^{+}(t) C_{\mu m^{\prime \prime} \sigma^{\prime}}(t), C_{\lambda m \sigma}^{+}(0) C_{\lambda m \sigma}(0)\right]\right\} \\
K_{2}\left(\mu \lambda \lambda^{\prime} m m^{\prime} m^{\prime \prime} \sigma \sigma^{\prime} \mid t\right)=-i \theta(t)\left\{\operatorname{Tr} \rho_{0}\left[C_{\mu m^{\prime} \sigma^{\prime}}^{+}(t) C_{\mu m^{\prime \prime} \sigma^{\prime}}(t), C_{\lambda m \sigma}^{+}(0) C_{\lambda^{\prime} m \sigma}(0)\right]\right\}
\end{gathered}
$$

where

$$
\left\{\begin{array}{rrr}
\theta(t) & =0 & t<0 \\
& =1 & t>0
\end{array}\right.
$$

The physical meaning of these response functions is very clear :

- $K_{1}$ expresses the response of the system measured at site $\mu$ and time $t$, involving both $\mid m^{\prime}>$ and $\left|m^{\prime \prime}\right\rangle$ type wave functions with spin $\sigma^{\prime}$, to perturbation applied at time 0 to the crystal field integrals corresponding to site $\lambda, \mid m>$ type wave functions and spin $\sigma$,

- $K_{2}$ expresses the similar response to perturbation applied in similar circumstances to the transfer integrals corresponding to site $\lambda$ and $\lambda^{\prime}$. Now the problem is to deal with the calculation of $K_{1}$ and $K_{2}$.

3. Properties of the response functions. - If we introduce the Fourier transforms of the response functions [12], i. e. :

$$
K_{1}(\omega)=\frac{1}{2 \pi} \int_{-\infty}^{+\infty} K_{1}(t) \mathrm{e}^{i \omega t} \mathrm{~d} t
$$

It can be shown that

$$
K_{1}(\omega)=\lim _{\eta \rightarrow 0^{+}} \int_{-\infty}^{+\infty} \frac{J_{1}\left(\omega^{\prime}\right) \mathrm{d} \omega^{\prime}}{\omega-\omega^{\prime}+i \eta}
$$

with

$$
\begin{aligned}
J_{1}\left(\omega^{\prime}\right)=\frac{1}{2 \pi} \sum_{a, b} \exp \frac{\left(\Omega+\mu N_{b}-E_{b}\right)}{k_{\mathrm{B}} t}<a \mid C_{\mu m^{\prime} \sigma^{\prime}}^{+} & C_{\mu m^{\prime \prime} \sigma^{\prime}} \mid b>\times \\
& \times<b\left|C_{\lambda m \sigma}^{+}(0) C_{\lambda m \sigma}(0)\right| a>\left[\exp \left(\frac{\omega}{k_{\mathrm{B}} t}\right)-1\right] \delta\left(\omega-\varpi_{b a}\right)
\end{aligned}
$$

where $|a\rangle$ and $|b\rangle$ are eigen states of $\mathscr{K}_{0}, \Omega$ is the termodynamical potential of the system, $\mu$ the chemical potential and $\omega_{b a}=E_{b}-E_{a}-\mu\left(N_{b}-N_{a}\right)\left(E_{a}\right.$ and $N_{a}$ being the energy and the particule number in $|a\rangle$ state).

It is then interesting [13] to introduce the functions :

$$
\begin{array}{rlr}
\tilde{K}_{1}(\omega) & =-\operatorname{Tr}\left[\rho_{0} \mathrm{e}^{+H_{0} \tau} C_{\mu m^{\prime} \sigma^{\prime}}^{+} C_{\mu m^{\prime \prime} \sigma^{\prime}} \mathrm{e}^{-H_{0} \tau} \hat{n}_{\lambda m \sigma}\right] & \text { if } \tau>0 \\
& =-\operatorname{Tr}\left[\rho_{0} \mathrm{e}^{-H_{0} \tau} \hat{n}_{\lambda m \sigma} \mathrm{e}^{+H_{0} \tau} C_{\mu m^{\prime} \sigma^{\prime}}^{+} C_{\mu m^{\prime \prime} \sigma^{\prime}}\right] & \text { if } \tau<0
\end{array}
$$

where $H_{0}=H_{0}-\mu N$.

As $\widetilde{K}_{1}$ can be written

$$
\tilde{K}_{1}\left(\omega_{n}\right)=\int_{-\infty}^{+\infty} \frac{\tilde{J}_{1}\left(\omega^{\prime}\right)}{i \omega_{n}-\omega^{\prime}} \mathrm{d} \omega^{\prime}
$$

it is clear that $K_{1}(\omega)$ can be considered as an analytic continuation of $\widetilde{K}_{1}\left(\omega_{n}\right)$ when $i \omega_{n} \rightarrow \omega+i \eta$. This is a very interesting remark because $\widetilde{K}_{1}\left(\omega_{n}\right)$ can be calculated through eq. (8) by using the powerful Green function and diagram methods at $T=0 \mathrm{~K}[13]$.

Additional simplifying hypotheses over the one-electron thermodynamical Green functions will be introduced as the following:

- they have only diagonal elements

$$
\mathfrak{S}_{\lambda \lambda^{\prime} m m^{\prime} \sigma \sigma^{\prime}}=\delta_{m m^{\prime}} \delta_{\sigma \sigma^{\prime}} \mathfrak{G}_{\lambda \lambda^{\prime} m m \sigma \sigma}
$$


- they can be calculated from the unperturbed Hamiltonian $\mathcal{H}_{0}$

$$
\mathcal{S}_{\lambda \lambda^{\prime} m m \sigma \sigma}=\mathcal{G}_{\lambda \lambda^{\prime} \sigma}
$$

- the electron-electron interactions are restricted to the intraatomic ones.

Then, in the random phase approximation the $\widetilde{K}_{1}(\omega)$ functions are solutions of a classicall Bethe ${ }_{-}^{\tau}$ Salpeter equation (formaly equivalent to the Dyson's equation $G=G_{0}+G_{0} V G$ ):

$$
\begin{aligned}
& \widetilde{K}_{1}\left(\mu \lambda m m^{\prime} m^{\prime \prime} \sigma \sigma^{\prime} \mid \omega_{n}\right)=\widetilde{\Pi}_{0}\left(\mu \lambda \sigma \mid \omega_{n}\right) \delta_{m m^{\prime}} \delta_{m m^{\prime \prime}} \delta_{\sigma \sigma^{\prime}} \\
& \quad+\sum_{\lambda^{\prime} m^{3} m^{4} \sigma^{\prime \prime}} \tilde{\Pi}_{0}\left(\mu \lambda^{\prime} \sigma \mid \omega_{n}\right) U^{\lambda^{\prime}}\left(m^{\prime} m^{\prime \prime} m^{3} m^{4} \sigma^{\prime} \sigma^{\prime \prime}\right) \widetilde{K}_{1}\left(\lambda^{\prime} m^{3} m^{4} \sigma^{\prime \prime} \lambda m \sigma \mid \omega_{n}\right)
\end{aligned}
$$

where $U^{\lambda^{\prime}}$ is the matrix element of the electron-electron interaction at site $\lambda^{\prime}$.

$\widetilde{\Pi}_{0}$ is the polarization function (or electron-hole bubble).

In the random phase approximation $U^{\lambda^{\prime}}$ does not depend on $\omega$ and :

$$
\tilde{\Pi}_{0}=k_{\mathrm{B}} T \sum_{\omega v} \mathcal{G}_{\mu \lambda \sigma}\left(\omega_{v}\right) \mathcal{G}_{\lambda \mu \sigma}\left(\omega_{v}+\omega_{n}\right)
$$

with

$$
\omega_{v}=(2 v+1) \pi k_{\mathrm{B}} T .
$$

A very similar treatment from the second eq. (6) leads to the expression whose solutions give the $\widetilde{K}_{2}\left(\omega_{n}\right)$ functions :

$\tilde{K}_{2}\left(\mu \lambda \lambda^{\prime} m m^{\prime} m^{\prime \prime} \sigma \sigma^{\prime} \mid \omega_{n}\right)=\widetilde{\Pi}_{1}\left(\mu \lambda \lambda^{\prime} \sigma \mid \omega_{n}\right) \delta_{m m^{\prime}} \delta_{m m^{\prime \prime}} \delta_{\sigma \sigma^{\prime}}$

$$
+\sum_{\lambda^{\prime \prime} m^{3} m^{4} \sigma^{\prime \prime}} U^{\lambda^{\prime \prime}} \tilde{\Pi}_{1}\left(\lambda \lambda^{\prime} \lambda^{\prime \prime} \sigma \mid \omega_{n}\right) \tilde{K}_{1}\left(\mu \lambda^{\prime \prime} m^{\prime} m^{\prime \prime} m^{3} m^{4} \sigma \mid \omega_{n}\right)
$$

with

$$
\widetilde{\Pi}_{1}=k_{\mathrm{B}} T \sum_{\omega \nu} \mathcal{G}_{\mu \lambda \sigma}\left(\omega_{v}\right) \mathbf{S}_{\lambda^{\prime} \mu \sigma}\left(\omega_{v}+\omega_{n}\right)
$$

Now, to go further into the calculation of $\langle\hat{A}(t)\rangle$ through eq. (5) we need more details regarding the physical system, i. e. the metal or alloy, which is involved. This will be the purpose of the next sections.

4. Calculation of the local EFG in pure non-cubic transition metals. - 4.1 THE EXPRESSION OF THE RESPONSE FUNCTIONS. - 4.1.1. In a pure metal, which remains invariant by translation through the lattice, some interesting simplifications can be carried out in the calculation of the response functions, resulting from the following translational properties :

$$
\widetilde{K}_{1}(\mu, \lambda)=\widetilde{K}_{1}(0, \lambda-\mu)=\widetilde{K}_{1}(\mu-\lambda, 0)
$$

whose common value will be noted

$$
\widetilde{K}_{1}\left(\mu^{\prime}\right) \quad \text { with } \quad \mathbf{R}_{\mu^{\prime}}=\mathbf{R}_{\lambda}-\mathbf{R}_{\mu}
$$

$\widetilde{K}_{2}, \widetilde{\Pi}_{0}$ and $\widetilde{\Pi}_{1}$ have similar translational properties.

4.1.2. The electron-electron interaction can be reasonably expressed using the usual Dworin and Narath approximation [14] as we have explained earlier [3], so that :

$$
\begin{array}{rlrl}
U^{\lambda}\left(m m^{\prime} m^{\prime \prime} m^{3} \sigma-\sigma\right) & =\delta_{m m^{\prime}} \delta_{m^{\prime \prime} m^{3}} U & & \text { (if } \left.m \neq m^{\prime \prime}\right) \\
U^{\lambda}\left(m m^{\prime} m^{\prime \prime} m^{3} \sigma \sigma\right) & =\delta_{m m^{\prime}} \delta_{m^{\prime \prime} m^{3}}(U-J) & & \text { (if } \left.m \neq m^{\prime \prime}\right) \\
& =0 & & \text { (if } \left.m=m^{\prime \prime}\right) \\
U^{\lambda}(m m m m \sigma-\sigma) & =U+J &
\end{array}
$$

where $U$ and $J$ are respectively the Coulomb and exchange interaction between $\mid m \sigma>$ states. 
As these matrix elements are not site dependent, eq. (9) giving $\widetilde{K}_{1}$ can be rewritten in a straightforward manner :

$$
\widetilde{K}_{1}\left(\mu m m^{\prime} m^{\prime \prime} \sigma \sigma^{\prime} \mid \omega_{n}\right)=0 \quad\left(\text { If } m^{\prime} \neq m^{\prime \prime}\right)
$$

and

$\tilde{K}_{1}\left(\mu m m^{\prime} \sigma \sigma^{\prime} \mid \omega_{n}\right)=\tilde{\Pi}_{0}\left(\mu \sigma^{\prime} \mid \omega_{n}\right) \delta_{m m^{\prime}} \delta_{\sigma \sigma^{\prime}}+\sum_{\lambda} \tilde{\Pi}_{0}\left(\lambda, \sigma^{\prime} \mid \omega_{n}\right) \times$

$$
\begin{aligned}
& \left\{(U-J) \sum_{m^{\prime \prime \neq m^{\prime}}} \widetilde{K}_{1}\left(m^{\prime \prime} \sigma_{1}(\mu-\lambda) m \sigma \mid \omega_{n}\right)+U \sum_{m^{\prime \prime} \neq m^{\prime}} \widetilde{K}_{1}\left(m^{\prime \prime}-\sigma^{\prime}(\mu-\lambda) m \sigma \mid \omega_{n}\right)\right. \\
& \left.\quad(U+J) \widetilde{K}_{1}\left(m^{\prime}-\sigma^{\prime}(\mu-\lambda) m \sigma \mid \omega_{n}\right)\right\}
\end{aligned}
$$

The lattice translational invariance suggests to substitute in eq. (11) the spatial Fourier transforms of $\widetilde{\Pi}_{0}$ and $\widetilde{K}_{1}$ i. e. :

$$
\begin{gathered}
\tilde{\Pi}_{0}\left(\mathbf{q}, \sigma^{\prime} \mid \omega_{n}\right)=\frac{1}{N} \sum_{\mu} \widetilde{\Pi}_{0}\left(\mu, \sigma \mid \omega_{n}\right) \exp (i \mathbf{q} \mathbf{R} \mu) \\
\widetilde{K}_{1}\left(\mathbf{q}, m m^{\prime} \sigma \sigma^{\prime} \mid \omega_{n}\right)=\frac{1}{N} \sum_{\mu} \widetilde{K}_{1}\left(\mu m m^{\prime} \sigma \sigma^{\prime} \mid \omega_{n}\right) \exp (i \mathbf{q} \mathbf{R} \mu)
\end{gathered}
$$

which in abridged notations leads to :

$$
\begin{aligned}
\tilde{K}_{1}\left(\mathbf{q} \mid \omega_{n}\right)=\tilde{\Pi}_{0}\left(\mathbf{q} \mid \omega_{n}\right)\left\{\delta_{m m^{\prime}} \delta_{\sigma \sigma^{\prime}}+(U-J)\right. & \sum_{m^{\prime \prime} \neq m^{\prime}} \widetilde{K}_{1}\left(\mathbf{q}, \sigma^{\prime} \mid \omega_{n}\right)+ \\
& \left.\quad U \sum_{m^{\prime \prime} \neq m^{\prime}} \widetilde{K}_{1}\left(\mathbf{q},-\sigma^{\prime} \mid \omega_{n}\right)+(U+J) \widetilde{K}_{1}\left(\mathbf{q},-\sigma^{\prime}, m^{\prime} \mid \omega_{n}\right)\right\} .
\end{aligned}
$$

Now, the initial linear system (11) of $4 N(2 l+1)^{2}$ equations reduces to $4(2 l+1)^{2}$ ones (where $l$ refers to

\begin{tabular}{|c|c|c|}
\hline$m, m^{\prime}, \sigma, \sigma^{\prime}$ & $\tilde{K}_{1}$ & Short notations \\
\hline 一 & - & - \\
\hline $\begin{array}{l}m=m^{\prime} \\
\sigma_{.}=\sigma^{\prime}=\uparrow \text { or } \downarrow\end{array}$ & $\frac{\widetilde{\Pi}_{0}^{\dagger \circ r \downarrow}}{D}\left\{1+(U-J) \tilde{\Pi}_{0}^{\downarrow}+\tilde{F}_{\text {for } \downarrow}^{+}\right\}$ & $\tilde{x}_{1}$ or $\tilde{y}_{1}$ \\
\hline $\begin{array}{l}m=m^{\prime} \\
\sigma=\uparrow \text { or } \downarrow \\
\sigma^{\prime}=\downarrow \text { or } \uparrow\end{array}$ & $\frac{J}{D}\left\{\tilde{\Pi}_{0}^{\dagger} \tilde{\Pi}_{0}^{\downarrow}+\tilde{\Pi}_{0}^{\dagger} \widetilde{F}_{\text {lor } \uparrow}\right\}$ & $\tilde{x}_{2}$ or $\tilde{y}_{2}$ \\
\hline $\begin{array}{l}m \neq m^{\prime} \\
\sigma=\uparrow \text { or } \downarrow \\
\sigma^{\prime}\end{array}$ & $\frac{\tilde{\Pi}_{0}^{\text {for } \downarrow} \tilde{F}_{\mathrm{o}^{\prime}}^{\text {or }}-}{D}$ & $\tilde{x}_{3}^{\sigma^{\prime}}$ or $\tilde{y}_{3}^{\sigma^{\prime}}$ \\
\hline
\end{tabular}
the band) whose solutions are presented in table I. As the $m$ dependence of $\widetilde{K}_{1}$ is restricted to the $\delta_{m m}$, term, there are only few different solutions.

The calculation of the $\widetilde{K}_{2}$ response function is quite similar and the results are presented in table II.

In both tables I and II suitable abridged notations have been introduced for convenience :

\section{TABLE I}

$$
\widetilde{K}_{1}\left(q m m^{\prime} \sigma \sigma^{\prime} \mid \omega_{n}\right) \text { values }
$$


TABle II

$\widetilde{K}_{2}\left(q m m^{\prime} \sigma \sigma^{\prime} \lambda \mid \omega_{n}\right)$ values

$$
\begin{aligned}
& \begin{array}{cc}
m m^{\prime} \sigma \sigma^{\prime} & \tilde{K}_{2} \\
- & -
\end{array} \\
& m=m^{\prime} \\
& \sigma=\sigma^{\prime}=\uparrow \\
& \tilde{\Pi}_{1}^{\uparrow}\left\{1+(U+J) \tilde{x}_{2}+2 l(U-J) \tilde{x}_{3}^{\uparrow}+2 l U \tilde{x}_{3}^{\downarrow}\right\} \\
& m=m^{\prime} \\
& \sigma=\sigma^{\prime}=\downarrow \\
& \tilde{\Pi}_{1}^{\downarrow}\left\{1+(U+J) \tilde{y}_{2}+2 l(U-J) \tilde{y}_{3}^{\downarrow}+2 l U \tilde{y}_{3}^{\uparrow}\right\} \\
& m=m^{\prime} \\
& \sigma=\uparrow \\
& \sigma^{\prime}=\downarrow \\
& m=m^{\prime} \\
& \sigma=\downarrow \\
& \sigma^{\prime}=\uparrow \\
& m \neq m^{\prime} \\
& \sigma=\sigma^{\prime}=\uparrow \\
& \tilde{\Pi}_{1}^{\downarrow}\left\{(U+J) \tilde{x}_{1}+2 l(U-J) \tilde{x}_{3}^{\downarrow}+2 l U \tilde{x}_{3}^{\uparrow}\right\} \\
& \tilde{\Pi}_{1}^{\uparrow}\left\{(U+J) \tilde{y}_{1}+2 l(U-J) \tilde{y}_{3}^{\dagger}+2 l U y_{3}^{\downarrow}\right\} \\
& \tilde{\Pi}_{1}^{\uparrow}\left\{(U-J) \tilde{x}_{1}+U \tilde{x}_{2}+(2 l-1) \tilde{x}_{3}^{\dagger}(U-J)+2(l U+J) \tilde{x}_{3}^{\downarrow}\right\} \\
& m \neq m^{\prime} \\
& \sigma=\uparrow \\
& \sigma^{\prime}=\downarrow \\
& m \neq m^{\prime} \\
& \sigma=\sigma^{\prime}=\downarrow \\
& m \neq m^{\prime} \\
& \sigma=\downarrow \\
& \sigma^{\prime}=\uparrow \\
& \tilde{\Pi}_{1}^{\downarrow}\left\{(U-J) \tilde{x}_{2}+U \tilde{x}_{1}+(2 l-1)(U-J) \tilde{x}_{3}^{\downarrow}+2(l U+J) \tilde{x}_{3}^{\uparrow}\right\} \\
& \widetilde{K}_{2}(4, \uparrow) \\
& \widetilde{K}_{2}(3, \downarrow) \\
& \tilde{\Pi}_{1}^{\downarrow}\left\{(U-J) \tilde{y}_{1}+U \tilde{y}_{2}+(2 l-1)(U-J) \tilde{y}_{3}^{\downarrow}+2(l U+J) \tilde{y}_{3}^{\dagger}\right\} \\
& \tilde{\Pi}_{1}^{\uparrow}\left\{(U-J) \tilde{y}_{2}+U \tilde{y}_{1}+(2 l-1)(U-J) \tilde{y}_{3}^{\uparrow}+2(l U+J) \tilde{y}_{3}^{\downarrow}\right\} \\
& \tilde{\Pi}_{0}^{\text {†or } \downarrow}=\widetilde{\Pi}_{0}\left(\mathbf{q}, \sigma=\uparrow \text { or } \downarrow \mid \omega_{n}\right) \\
& D=\left[1+(U-J) \tilde{\Pi}_{0}^{\dagger}\right]\left[1+(U-J) \tilde{\Pi}_{0}^{\downarrow}-J^{2} \tilde{\Pi}_{0}^{\dagger} \tilde{\Pi}_{0}^{\downarrow}\right] \\
& \tilde{F}_{\sigma}^{+}=(U-J) X_{\sigma}+\left(U^{2}-J^{2}\right) \tilde{\Pi}_{0}^{\downarrow} Y_{\sigma}+\left(U^{2}+J^{2}-U J\right) X_{\sigma} \tilde{\Pi}_{0}^{\dagger} \\
& \tilde{F}_{\sigma}^{-}=(U-J) Y_{\sigma}+\left(U^{2}-J^{2}\right) \tilde{\Pi}_{0}^{\dagger} X_{\sigma}+\left(U^{2}+J^{2}-U J\right) Y_{\sigma} \tilde{\Pi}_{0}^{\downarrow} \\
& X_{\sigma}=\frac{\tilde{\Pi}_{0}^{1}}{\Delta}\left[\delta_{\uparrow \sigma}\left\{1-2 l(U-J) \tilde{\Pi}_{0}^{\downarrow}\right\}+(2 l+1)(U+J) \tilde{\Pi}_{0}^{\downarrow} \delta_{\downarrow \sigma}\right] \\
& Y_{\sigma}=\frac{\tilde{\Pi}_{0}^{\downarrow}}{\Delta}\left[\delta_{\downarrow \sigma}\left\{1-2 l(U-J) \tilde{\Pi}_{0}^{\uparrow}\right\}+(2 l+1)(U+J) \tilde{\Pi}_{0}^{\uparrow} \delta_{\uparrow \sigma}\right] \\
& \Delta=\left[1-2 l(U-J) \tilde{\Pi}_{0}^{\dagger}\right]\left[1-2 l(U-J) \tilde{\Pi}_{0}^{\downarrow}\right]-[(2 l+1)(U+J)]^{2} \tilde{\Pi}_{0}^{\uparrow} \tilde{\Pi}_{0}^{\downarrow} .
\end{aligned}
$$

The analytic continuations of $\widetilde{K}_{1}$ and $\widetilde{K}_{2}$ to obtain the true response functions $K_{1}$ and $K_{2}$ as explained in section 3 can be made merely on $\Pi_{0}$ and $\Pi_{1}$ since $\widetilde{K}_{1}$ and $\widetilde{K}_{2}$ are expressed in terms of $\Pi_{0,1}^{\text {tort }}$ and $U$ and $J$.

4.2 MeAN VALUE $\langle\hat{A}(t)\rangle$ OF THE ANISOTROPIC OPERATOR. - The mean value $\langle\hat{A}(t)\rangle$ is expressed through eq. (5) which can be rewritten by expanding $\langle\hat{A}(t)\rangle, V_{m}^{\lambda}$ and $\Delta T_{m}^{\lambda \lambda^{\prime}}$ in time Fourier transforms

$$
\begin{aligned}
& \langle\hat{A}(\omega)\rangle=\frac{1}{2 \pi} \int_{-\infty}^{+\infty}\left\langle\hat{A}(t)>\mathrm{e}^{i \omega t} \mathrm{~d} t\right. \\
& V_{m}^{\lambda}(\omega) \quad=\frac{1}{2 \pi} \int_{-\infty}^{+\infty} V_{m}^{\lambda}(t) \mathrm{e}^{i \omega t} \mathrm{~d} t=V_{m}(\omega) \\
& \Delta T_{m}^{\lambda \lambda^{\prime}}(\omega)=\frac{1}{2 \pi} \int_{-\infty}^{+\infty} \Delta T_{m}^{\lambda \lambda^{\prime}}(t) \mathrm{e}^{i \omega t} \mathrm{~d} t=\Delta T_{m}^{\lambda}(\omega) .
\end{aligned}
$$


The $\left\langle A^{0}\right\rangle$ value at the origin site can then be obtained in a straightforward manner, leading to

$$
\left\langle A^{0}(\omega)\right\rangle=\left\langle A_{1}^{\mathrm{o}}(\omega)\right\rangle+\left\langle A_{2}^{\mathrm{o}}(\omega)\right\rangle
$$

with

$$
\left.\begin{array}{c}
<A_{1}^{0}(\omega)>=-\chi_{q}^{1}(\omega) \sum_{m} V_{m}(\omega) A_{m m}^{0} \\
<A_{2}^{0}(\omega)>=-\left(\sum \chi_{q}^{2}(\lambda, \omega)\right)\left(\sum_{m} A_{m m}^{0} \Delta T_{m}^{\lambda}(\omega)\right)
\end{array}\right\}
$$

$\chi_{q}^{1}(\omega)$ and $\chi_{q}^{2}(\lambda, \omega)$ are two susceptibility functions expressed by :

$$
\begin{aligned}
\chi_{q}^{1}(\omega) & =-\frac{1}{D}\left(\Pi_{0}^{\uparrow}+\Pi_{0}^{\downarrow}+2 U \Pi_{0}^{\uparrow} \Pi_{0}^{\downarrow}\right) \\
\chi_{q}^{2}(\lambda, \omega) & =-\frac{1}{D}\left[\Pi_{1}^{\uparrow}+\Pi_{1}^{\downarrow}+U\left(\Pi_{1}^{\uparrow} \Pi_{0}^{\downarrow}+\Pi_{1}^{\downarrow} \Pi_{0}^{\uparrow}\right)\right] .
\end{aligned}
$$

The most simple way to obtain $\Pi_{0}$ and $\Pi_{1}$ using eq. $(9 \mathrm{bis})$ and (10 bis) respectively is to consider only Green functions without interactions; this gives :

$$
\tilde{\Pi}_{0}(\mathbf{q}, \sigma \mid \omega)=-\chi_{0}^{\sigma}(\mathbf{q}, \omega)
$$

and

$$
\begin{aligned}
\tilde{\Pi}_{1}(\mathbf{q}, \lambda, \sigma \mid \omega) & =-\frac{1}{N} \sum_{\mathbf{k}} \frac{\left[f\left(\varepsilon_{k}^{\sigma}\right)-f\left(\varepsilon_{k+q}^{\sigma}\right)\right] \mathrm{e}^{i \mathbf{k} \cdot \mathbf{R}_{\lambda}}}{\varepsilon_{k+q}^{\sigma}-\varepsilon_{k}^{\sigma}+\omega+i \eta} \\
& =-\frac{1}{N} \sum_{k} g^{\sigma}(\mathbf{k}, \mathbf{q}, \omega) \exp \left(i \mathbf{k} \mathbf{R}_{\lambda}\right)
\end{aligned}
$$

where $\chi_{0}^{\sigma}$ is the Pauli band susceptibility and $f(\varepsilon)$ the Fermi distribution function. It may be worth noting that $\chi_{q}^{1}(\omega)$ and $\chi_{q}^{2}(\omega)$ can be really used to obtain the mean value of any operator which would be sensitive to the material anisotropy through partly localised electrons (EFG, electrical resistivity, magnetic susceptibility...).

4.3 SIMPLIFIED EXPRESSION FOR THE LOCAL EFG. - To obtain the localized contribution $e q_{\text {Loc }}$ to the EFG eq. (13) can be used by substituting the true expression of the quadrupole operator

$$
\widehat{A}=-e \frac{2 P_{2}^{0}(\cos \theta)}{r^{3}}
$$

in the summations $\sum_{m} V_{m}(\omega) A_{m m}$ and $\sum_{m} \Delta T_{m}^{\lambda}(\omega) A_{m m}$.

The former does not depend on the band structure and corresponds only to the influence of the anisotropy on the sub-band position ; it can be easily evaluated if the crystal potential is expressed by the spherical harmonic addition theorem. The later is in fact a summation of $\mathbf{k}$ dependent terms each of which corresponding to the energy change $\delta E_{m}(\mathbf{k})$ at wave vector $\mathbf{k}$ in sub-band $m$.

Finally, $e q_{\mathrm{Loc}}$ can be expressed as

$$
e q_{\mathrm{Loc}}=e q_{1}+e q_{2}
$$

with

$$
\left.\begin{array}{l}
e q_{1}=-2 \frac{e^{2}}{5} \frac{r^{-3}><r^{2}>(2 l+1)}{(2 l-1)(2 l+3)}\left(\frac{\mathrm{d} P_{l}^{0}(u)}{\mathrm{d} u}\right)_{u=1} e q_{\mathrm{NLoc}} \chi_{q}^{1}(\omega) \\
e q_{2}=-\left\{\sum_{m}<e q>_{m} \sum_{\mathbf{k}} \delta E_{m}(\mathrm{k})\right\} \chi_{q}^{2}(\omega)
\end{array}\right\}
$$

where $P_{l}^{0}(u)$ is Legendre polynomial of $u, e q_{\mathrm{NLoc}}$ is the lattice contribution to the EFG and

$$
\delta E_{m}(\mathbf{k})=\sum_{\lambda \neq 0} \Delta T_{m}^{\lambda} \exp \left(i \mathbf{k} \mathbf{R}_{\lambda}\right) .
$$


$\chi_{q}^{1}$ and $\chi_{q}^{2}$ are given by eq. (14) and (15) which leads to :

$$
\left.\begin{array}{l}
\chi_{q}^{1}(\omega)=\frac{\chi_{0}^{\uparrow}(\omega)+\chi_{0}^{\downarrow}(\omega)-2 U \chi_{0}^{\dagger}(\omega) \chi_{0}^{\downarrow}(\omega)}{\left[1-(U-J) \chi_{0}^{\uparrow}(\omega)\right]\left[1-(U-J) \chi_{0}^{\downarrow}(\omega)\right]-J^{2} \chi_{0}^{\dagger}(\omega) \chi_{0}^{\downarrow}(\omega)} \\
\chi_{q}^{2}(\omega, \mathbf{k})=\frac{g \uparrow(\mathbf{k}, 0, \omega)+g \downarrow(\mathbf{k}, 0, \omega)-U\left[g \uparrow(\mathbf{k}, 0, \omega) \chi_{0}^{\downarrow}(\omega)+g \downarrow(\mathbf{k}, 0, \omega) \chi_{0}^{\dagger}(\omega)\right]}{\left[1-(U-J) \chi_{0}^{\uparrow}(\omega)\right]\left[1-(U-J) \chi_{0}^{\downarrow}(\omega)\right]-J^{2} \chi_{0}^{\dagger}(\omega) \chi_{0}^{\downarrow}(\omega)}
\end{array}\right\} .
$$

(For a paramagnetic $3 \mathrm{~d}$ transition material, at $T=0 \mathrm{~K}$ and in the static approximation $\omega=0, e q_{1}$ reduces to the expression of $e q_{\text {Loc }}$ obtained in the Hartree-Fock approximation [3]). To go furtber into detailed calculation needs both a precise description of the material structure and extra simplification in the evaluation of the $\delta E_{m}(\mathbf{k})$ quantities.

5. Localized contribution to the EFG on transition impurities in non-cubic metals. - 5.1 TRANSITION IMPURITY IN NORMAL METALS. - Assuming that the direct influence of the crystal field on the host $s$-band is not too strong, the perturbation potential of a single transition impurity can furthermore be assumed mostly localized in the center of the impurity cell itself. Then, the perturbation part of the Hamiltonian (eq. 2) can be reasonably reduced to :

$$
\mathfrak{H}_{1}=\sum_{m \sigma} V_{m}^{0} C_{m \sigma 0}^{0} C_{m \sigma 0}
$$

where 0 refers to the impurity site.

So the influence of the anisotropy results only in changes for the positions of the impurity electronic levels and $e q_{\text {Loc }}$ can be merely identified to $e q_{1}$ (eq. 16) :

$$
e q_{\mathrm{Loc}}=-\frac{e^{2}}{5} \frac{r^{-3}><r^{2}>(2 l+1)}{(2 l-1)(2 l+3)}\left(\frac{\mathrm{d} P_{l}^{0}(u)}{\mathrm{d} u}\right)_{u=1} \chi_{q}^{1}(\omega) e q_{\mathrm{NLoc}}
$$

Again, this result is consistent with an Hartree-Fock calculation [3] in the limits $T \rightarrow 0 \mathrm{~K}$ and $\omega \rightarrow 0$.

5.2 TRANSITION IMPURITY IN TRANSITION METALS. - A calculation of the EFG, even restricted to the localized contribution, in the most general transition-transition system could be carried out in principle, starting as in the previous sections from the expressions of the responses functions given by eq. (9) and (10). However, the results reported here will deal only with rather simple dilute systems in which the substitutional impurity is so too far from the host in the periodic classification, so that the crystallographic point group of the host can be considered as not altered by the impurity presence and that both host and impurity atoms have electrons with the same l-like orbital character.

Assuming furthermore, after Lederer and Mills [15], that the impurity influence on the electron-hole bubble $\widetilde{\Pi}_{0}(q \mid \omega)$ is negligible, the impurity effect on the band host will mostly result in changes $\Delta U=U_{i}-U_{0}$ and $\Delta J=J_{i}-J_{0}$ for the Coulomb and exchange integrals at the impurity site.

So, eq. (11) and (12) can be straightforward rewritten by substituting $U_{0}$ and $J_{0}$ to $U$ and $J$ for all sites but the impurity one for which $U$ and $J$ must be changed into $U_{0}+\Delta U$ and $J_{0}+\Delta J$ respectively. This results in the appearance of four more terms in the right parts of eq. (11) and (12) which correspond to the perturbation introduced by the impurity, and as a consequence the response functions as well as the EFG will be different at the impurity site with respect to a normal host site.

For instance, if we concentrate for simplicity's sake on paramagnetic systems where $\widetilde{K}_{1}$ and $\widetilde{K}_{2}$ are not spin dependent, $\widetilde{K}_{1}$ are now solutions of the following set of equations, quite formaly similar to eq. (12) :

$$
\begin{aligned}
\widetilde{K}_{1}\left(\mathbf{q} \mathbf{q}^{\prime} m m^{\prime} \mid \omega_{n}\right)=\widetilde{\Pi}^{0}\left(\mathbf{q} \mid \omega_{n}\right)\left\{\delta\left(\mathbf{q}-\mathbf{q}^{\prime}\right)\right. & \delta_{m m^{\prime}}+\left(2 U_{0}-J_{0}\right) \sum_{m^{\prime \prime} \neq m} \widetilde{K}_{1}\left(\mathbf{q} \mathbf{q}^{\prime} m m^{\prime \prime} \mid \omega_{n}\right)+ \\
& +\left(U_{0}+J_{0}\right) \widetilde{K}_{1}\left(\mathbf{q} \mathbf{q}^{\prime} m m^{\prime} \mid \omega_{n}\right)+\frac{1}{N}(2 \Delta U-\Delta J) \sum_{m^{\prime \prime} \neq m} \tilde{K}_{1}^{i}\left(\mathbf{q}^{\prime} m m^{\prime \prime} \mid \omega_{n}\right) \\
& \left.+\frac{\Delta U+\Delta J}{N} \widetilde{K}_{1}^{i}\left(\mathbf{q}^{\prime} m m^{\prime} \mid \omega_{n}\right)\right\}
\end{aligned}
$$

with

$$
\begin{aligned}
\widetilde{K}_{\mathbf{1}}\left(\mathbf{q q} \mathbf{q}^{\prime} m m^{\prime} \mid \omega_{n}\right)=\frac{1}{N} \sum_{\lambda, \mu} \exp \left[-i \mathbf{q}\left(\mathbf{R}_{\mu}-\mathbf{R}_{0}\right)\right] \exp \left[-i \mathbf{q}^{\prime}\left(\mathbf{R}_{\lambda}-\mathbf{R}_{0}\right)\right] \widetilde{K}_{1}\left(\mu \lambda m m^{\prime} \mid \omega_{n}\right) \\
K_{1}^{i}\left(\mathbf{q}^{\prime} m m^{\prime} \mid \omega_{n}\right)=\frac{1}{N} \sum_{\lambda} \exp \left[-i \mathbf{q}^{\prime}\left(\mathbf{R}_{\lambda}-\mathbf{R}_{0}\right)\right] \widetilde{K}_{1}\left(0 \lambda m m^{\prime} \mid \omega_{n}\right)
\end{aligned}
$$


where index 0 refers to the impurity site. A similar set of equations, obtained from eq. (10) leads to the $\widetilde{K}_{2}$ response functions. The results can be summarized as the following :

$$
\begin{aligned}
& \tilde{K}_{1}\left(\mathbf{q} \mathbf{q}^{\prime} m m^{\prime} \mid \omega_{n}\right)=-\tilde{\chi}_{q}^{1}\left(\mathbf{q} \mid \omega_{n}\right) \delta_{m m^{\prime}} \delta\left(\mathbf{q}-\mathbf{q}^{\prime}\right)+\tilde{H}\left(\mathbf{q}, \mathbf{q}^{\prime} \mid \omega_{n}\right) \\
& =\frac{\tilde{\chi}_{q}^{1}\left(\mathbf{q} \mid \omega_{n}\right) \tilde{\chi}_{q}^{1}\left(\mathbf{q}^{\prime} \mid \omega_{n}\right)}{N-(\Delta U-2 \Delta J) \sum_{\mathbf{q}} \tilde{\chi}_{q}^{1}\left(\mathbf{q} \mid \omega_{n}\right)}(\Delta U-2 \Delta J) \\
& \tilde{K}_{1}^{i}\left(\mathbf{q}^{\prime} m m^{\prime} \mid \omega_{n}\right) \quad=N \frac{\tilde{Y}_{i}\left(\mathbf{q}^{\prime} \mid \omega_{n}\right)-\tilde{\chi}_{q}^{1}\left(\mathbf{q}^{\prime} \mid \omega_{n}\right) \delta_{m m^{\prime}}}{N-(\Delta U-2 \Delta J) \sum_{\mathbf{q}} \tilde{\chi}_{q}^{1}\left(\mathbf{q} \mid \omega_{n}\right)}
\end{aligned}
$$

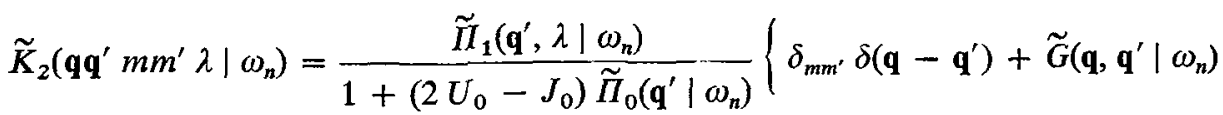

$$
\begin{aligned}
& \left.+\frac{(\Delta U-2 \Delta J) \delta_{i, j} \tilde{\chi}_{q}^{1}\left(\mathbf{q} \mid \omega_{n}\right)}{N-(\Delta U-2 \Delta J) \sum_{\mathbf{q}} \tilde{\chi}_{q}^{1}\left(\mathbf{q} \mid \omega_{n}\right)}\right\}
\end{aligned}
$$

with the definitions :

$$
\tilde{\chi}_{q}^{1}\left(\mathbf{q} \mid \omega_{n}\right)=\frac{-\tilde{\Pi}_{0}\left(\mathbf{q} \mid \omega_{n}\right)}{1+\left(U_{0}-2 J_{0}\right) \widetilde{\Pi}_{0}\left(\mathbf{q} \mid \omega_{n}\right)}
$$

(which is an anisotropic susceptibility):

$$
\begin{aligned}
& { }^{\prime} \tilde{H}\left(\mathbf{q q} \mathbf{q}^{\prime} \mid \omega_{n}\right)=-\tilde{\chi}_{q}^{1}\left(\mathbf{q} \mid \omega_{n}\right)\left\{\tilde{N}\left(\mathbf{q} \mathbf{q}^{\prime} \mid \omega_{n}\right) \cdot\left(2 U_{0}-J_{0}\right)+\tilde{N}_{i}\left(\mathbf{q}^{\prime} \mid \omega_{n}\right) \frac{(\Delta U-2 \Delta J)}{N}+\right. \\
& \left.+\frac{N \tilde{Y}_{i}\left(\mathbf{q}^{\prime} \mid \omega_{n}\right)}{N-(\Delta U-2 \Delta J) \sum_{\mathbf{q}} \tilde{\chi}_{q}^{1}\left(\mathbf{q} \mid \omega_{n}\right)}\right) \\
& \tilde{G}\left(\mathbf{q q ^ { \prime }} \mid \omega_{n}\right)=\widetilde{\Pi}_{1}\left(\mathbf{q}, \lambda \mid \omega_{n}\right)\left\{\left(2 U_{0}-J_{0}\right) \tilde{N}\left(\mathbf{q q ^ { \prime }} \mid \omega_{n}\right)-\left(U_{0}-2 J_{0}\right) \tilde{H}\left(\mathbf{q q ^ { \prime }} \mid \omega_{n}\right)+\right. \\
& \left.+\frac{(2 \Delta U-\Delta J)}{N} \tilde{N}_{i}\left(\mathbf{q}^{\prime} \mid \omega_{n}\right)-\frac{\Delta U-2 \Delta J}{N} \tilde{Y}_{i}\left(\mathbf{q}^{\prime} \mid \omega_{n}\right)\right\} \\
& \tilde{Y}_{i}\left(\mathbf{q}^{\prime} \mid \omega_{n}\right)=-\sum_{\mathbf{q}}\left\{\left(2 U_{0}-J_{0}\right) \tilde{\chi}_{q}^{1}\left(\mathbf{q} \mid \omega_{n}\right) \tilde{N}\left(\mathbf{q} \mathbf{q}^{\prime} \mid \omega_{n}\right)-\frac{(2 \Delta U-\Delta J)}{N} \tilde{N}_{i}\left(\mathbf{q}^{\prime} \mid \omega_{n}\right) \sum_{\mathbf{q}^{\prime}} \tilde{\chi}_{q}^{1}\left(\mathbf{q}^{\prime} \mid \omega_{n}\right)\right\} \\
& \tilde{N}\left(\mathbf{q} \mathbf{q}^{\prime} \mid \omega_{n}\right)=\tilde{F}\left(\mathbf{q} \mid \omega_{n}\right)\left\{\delta\left(\mathbf{q}-\mathbf{q}^{\prime}\right)+\frac{1}{N}[(4 l-1) \Delta U-2(l-1) \Delta J] \tilde{N}_{i}\left(\mathbf{q}^{\prime} \mid \omega_{n}\right)\right\} \\
& \tilde{N}_{i}\left(\mathbf{q}^{\prime} \mid \omega_{n}\right)=\frac{\tilde{F}\left(\mathbf{q}^{\prime} \mid \omega_{n}\right)}{1-[(4 l-1) \Delta U-2(l-1) \Delta J] \bar{F}\left(\omega_{n}\right)} \\
& \tilde{F}\left(\mathbf{q} \mid \omega_{n}\right)=\frac{\tilde{\Pi}_{0}\left(\mathbf{q} \mid \omega_{n}\right)}{1-\left[(4 l-1) U_{0}-(2 l-2) J_{0}\right] \tilde{\Pi}_{0}\left(\mathbf{q} \mid \omega_{n}\right)} \\
& \bar{F}\left(\omega_{n}\right) \quad=\frac{1}{N} \sum_{\mathbf{q}} \tilde{F}\left(\mathbf{q} \mid \omega_{n}\right)
\end{aligned}
$$

$\widetilde{\Pi}_{0}$ and $\widetilde{\Pi}_{1}$ are given by eq. (15) calculated in section 4.2 .

Using the same Fourier transform procedure as described in section 4.2 , it is now possible to obtain the mean value $\hat{A}(t)$, or, to be more precise, $A(\omega)$. The calculation is quite similar, regarding the formalism point of view, except that it is convenient to emphasize the site dependence of the crystal potential by writing its Fourier components :

$$
\begin{cases}V_{m}^{\lambda}(\omega)=V_{m}(\omega) & \text { if } \lambda=0 \\ V_{m}(\omega)=V_{m}(\omega)+\Delta V_{m}(\omega) & \text { if } \lambda=0\end{cases}
$$


which leads to :

$$
A(\omega)=\sum_{\lambda} A_{\lambda}(\omega)
$$

and :

$$
\left\langle A_{\lambda}(\omega)\right\rangle=\left\langle A_{\lambda^{1}}(\omega)\right\rangle+\left\langle A_{\lambda^{2}}(\omega)\right\rangle
$$

Assuming that the transfer integrals are not too much different from their values in the pure transition host material, $\left\langle A_{\lambda^{2}}(\omega)\right\rangle$ will be obtained in a good approximation using the second eq. (13) and the corresponding contribution to the localized EFG will be the same as $e q_{2}$ in eq. (16). So the presence of an impurity will introduce a change in the EFG on both itself and host atoms through the $e q_{1}$ contribution (as defined in section 4.3).

With the same notations, a straightforward calculation gives :

$$
\begin{aligned}
\left\langle A_{\lambda^{1}}(\omega)\right\rangle=-\chi_{q}^{1}(\omega)\left\{1+-\frac{(\Delta U-2 \Delta J) \chi_{q i}^{1}(\omega)}{1-(\Delta U-2 \Delta J) \overline{\chi_{a}^{1}}(\omega)}\right\} \sum_{m} V_{m}(\omega) A_{m m}^{\lambda} & \\
& -\frac{2 \chi_{q i}^{1}(\omega)}{1-(\Delta U-2 \Delta J) \overline{\chi_{q}^{1}}(\omega)} \sum_{m} \Delta V_{m}(\omega) A_{m m}^{\lambda}
\end{aligned}
$$

with

$$
\chi_{q i}^{1}(\omega)=\frac{1}{N} \sum_{\mathbf{q}} \chi_{q}^{\mathbf{1}}(\mathbf{q}, \omega) \mathrm{e}^{i \mathbf{q}\left(\mathbf{R}_{\lambda}-\mathbf{R}_{0}\right)} .
$$

The application of this result to EFG, as explained in section 4.3 gives the expression of $e q_{1}$ on both host and impurity site, i. e. :

$$
\left\{\begin{array}{c}
e q_{1}^{(\mathrm{H})}=e q_{1} \text { (pure host) }\left\{1+\frac{\Delta U+2 \Delta J \chi_{q i}^{1}(\omega)}{1-(\Delta U-2 \Delta J) \overline{\chi_{q}^{1}}(\omega)}\right\}- \\
-4 \frac{\mathrm{e}^{2}}{5} \frac{<r^{-3}>_{\mathrm{H}}\left[<r^{2}>_{i}-<r^{2}>_{\mathrm{H}}\right](2 l+1)}{(2 l-1)(2 l+3)} \frac{\chi_{q i}^{1}(\omega) e q_{\mathrm{NLoc}}}{1-(\Delta U-2 \Delta J) \overline{\chi_{q}^{1}}(\omega)} \\
e q_{1}^{(\mathrm{i})}=-4 \frac{e^{2}}{5} \frac{e r^{-3}>_{\mathrm{i}}(2 l+1)}{(2 l-1)(2 l+3)} \frac{e q_{\mathrm{NLoc}}}{1-(\Delta U-2 \Delta J) \chi_{q}^{1}(\omega)}\left\{<r^{2}>_{\mathrm{H}} \chi_{q}^{1}(\omega)+\right. \\
\left.+\left[<r^{2}>_{\mathrm{i}}-<r^{2}>_{\mathrm{H}}\right] \overline{\chi_{q}^{1}}(\omega)\right\}
\end{array}\right.
$$

Eq. (22a) contains some interesting features. In particular we see that the impurity introduces an extra contribution to the localized EFG on the host atoms; the magnitude of this extra contribution is mostly determined by $\chi_{q i}^{1}(\omega)$ which is the Fourier transform of the susceptibility (eq. (22)) and, as a consequence, is an oscillating function in space around the impurity. This new oscillating contribution is quite different from the classical Friedel oscillations [16]. The EFG which results from the classical Friedel oscillation arises from the anisotropy that may be created in the host metal by the impurity. The new oscillating term which appears in eq. (22a) is a response of the impurity to the actual pure host anisotropy.

6. Conclusion. - The dynamical theory which is presented in this paper is quite general and can be used to determine the anisotropy dependence of any physical parameter in a solid. The analytic expressions obtained for $\langle A(t)\rangle$ can be considered as without too much intricacies, particularly when applied to the calculation of the localized contribution to the EFG in non-cubic metals. The results obtained for pure transition metal, transition impurity in normal and transition metal cannot be extended to the calculation of the EFG in pure normal metals which are not well described in the tight binding approximation. It is worth emphasizing that the perhaps two main features of the calculations presented here were to treat the anisotropy influence as a perturbation term in the Hamiltonian of the system and to approach $\langle A(t)\rangle$ by linear response theory.

It will be the purpose of next papers to compare these theoretical results with experimental data, in particular with the view to present acceptable interpretations of sign, temperature and material dependence- of the EFG in hep systems, in connexion with some of their 
electronic properties (virtual bound states characteristics, Kondo temperature, magnetic transitions...). The systems which are relevant to our theory and for which valuable experimental data are available by now can be classified as the following :

1) Pure transition hep metals : Sc and $\mathrm{Ti} ; \mathrm{Y}, \mathrm{Zr}$,
Tc and $\mathrm{Ru} ; \mathrm{Hf}, \mathrm{Re}$ and $\mathrm{Os}$ in the $3 \mathrm{~d}, 4 \mathrm{~d}$ and $5 \mathrm{~d}$ series respectively,

2) Transition impurity in normal hcp metals : Fe, $\mathrm{Rh}$ and $\mathrm{Ta}$ as impurities in $\mathrm{Be}$ and $\mathrm{Zn}$,

3) Transition impurity in transition hcp metals : $\mathrm{Ta}$ and $\mathrm{Fe}$ as impurities in the first category elements, and $O$ s in $\mathrm{Re}$.

\section{References}

[1] Vianden, R., Proceeding of the International Meeting on Hyperfine Interaction, Louvain (1975).

[2] PIECuCH, M. and Janot, Ch., J. Physique Collog. 35 (1974) C 6-291.

[3] Pifcuch, M. and JANot, Ch., J. Phys. Chem. Solids 36 (1975) 1135

[4] Das, T. P., Phys. Script. 2 (1975) 121.

[5] NijBoer, B. R. A. and DE Wette, F. W., Physica 24 (1958) 422.

Pomerantz, M. and Das, T. P., Phys. Rev. 123 (1961) 2070.

[6] Simmons, W. W. and Slichter, C. P., Phys. Rev. 212 (1961) 1580.

[7] Quitmann, D., Nishiyama, K. and Rierel, D., Private communication. To be published.

[8] Lodge, K. W. and Sholl, C. A., J. Phys. F : Metal. Phys. 4 (1974) 2073.
[9] Mohapatra, N. C., Singal, C. N., Das, T. P. and Jena, P., Phys. Rev. Lett. 29 (1972) 456.

[10] Watson, R. E., Gossard, A. C. and Yaret, Y., Phys, Rev. A. 140 (1965) 375.

[11] Demangeat, C., J. Phys. F : Metal. Phys. 4 (1974) 164 and 5 (1975) 169.

[12] Zubarev, D. N., Sov. Phys. Usp. 3 (1960) 320.

[13] Abrikosov, A. A., Gorkov, L. P. and Dzialoshinski, I. E., Methods of quantum field theory in statistical Physics, Prentice Hall Inc. Englewood Cliffs, New York (1963).

[14] Dworin, L. and Narath, A., Phys. Rev. Lett. 25 (1970) 1287.

[15] Lederer, P. and Mrtus, O. L., Solid State Commun. 5 (1967) 131.

[16] Blandin, A. and Friedel, J., J. Phys. Rad. 23 (1962) 609. 